\title{
Pregnancy in Advanced Kidney Disease: Clinical Practice Considerations on a Challenging Combination
}

\author{
Rozemarijn Snoek $^{a, b} \quad$ Rieke van der Graaf ${ }^{c} \quad$ Jildau R. Meinderts ${ }^{d}$ \\ Franka van Reekum $^{\mathrm{e}}$ Kitty W.M. Bloemenkamp ${ }^{f} \quad$ Nine V.A.M. Knoers $^{\mathrm{a}} \mathrm{g}$ \\ Albertien M. van Eerde ${ }^{a, b} \quad$ A. Titia Lely ${ }^{f}$ \\ ${ }^{a}$ Department of Genetics, University Medical Center Utrecht, Utrecht University, Utrecht, The Netherlands; \\ ${ }^{b}$ Center for Molecular Medicine, Utrecht University, Utrecht, The Netherlands; 'Department of Medical Humanities, \\ Julius Center, University Medical Center Utrecht, Utrecht University, Utrecht, The Netherlands; ${ }^{d}$ Department \\ of Nephrology, University Medical Center Groningen, University of Groningen, Groningen, The Netherlands; \\ eDepartment of Nephrology, University Medical Center Utrecht, Utrecht University, Utrecht, The Netherlands; \\ fDepartment of Obstetrics, Birth Centre Wilhelmina Children's Hospital, University Medical Center Utrecht, Division \\ Women and Baby, Utrecht University, Utrecht, The Netherlands; ${ }^{9}$ Department of Genetics, University Medical Center \\ Groningen, University of Groningen, Groningen, The Netherlands
}

\author{
Keywords \\ Chronic kidney disease · Pregnancy · Genetic kidney \\ disease $\cdot$ Preimplantation genetic diagnostics
}

\begin{abstract}
Background: Thanks to the advances in care, pregnancy is now attainable for the majority of young female CKD patients, although it is still a high-risk endeavor. Clinical decision-making in these cases is impacted by a myriad of factors, making (pre)pregnancy counseling a complex process. The complexities, further impacted by limited data and unknown risks regarding outcome, can cause discussions when deciding on the best care for a specific patient. Objectives: In this article, we provide an overview of the considerations and dilemmas we encounter in preconception counseling and offer our perspective on how to deal with them in daily clinical practice. Methods: The main topics we discuss in our counseling are (1) the high risk of pregnancy complications, (2) the risk of permanent CKD deterioration due to pregnan-
\end{abstract}

\begin{tabular}{ll}
\hline karger@karger.com & (c) 2020 The Author(s) \\
www.karger.com/nef & Published by S. Karger AG, Basel \\
& This article is licensed under the Creative Commons Attribution- \\
Narger & Nonmercial-NoDerivatives 4.0 International License (CC BY- \\
NC-ND) (http://www.karger.com/Services/OpenAccessLicense). \\
Usage and distribution for commercial purposes as well as any dis- \\
tribution of modified material requires written permission.
\end{tabular}

cy and subsequent decreased life expectancy, (3) appropriate changes in renal medication, and (4) assisted reproduction, genetic testing, and prenatal or preimplantation genetic diagnostics. Results and Conclusions: In our clinic, we openly address moral dilemmas arising in clinical practice in pregnancy and CKD, both within the physician team and with the patient. We do this by ensuring an interpretive physician-patient interaction and shared decision-making, deliberating in a multidisciplinary setting and, if needed, with input from an expert committee. @ 2020 The Author(s)

Published by S. Karger AG, Basel

\section{Background}

Pregnancy is now attainable for a majority of young female CKD patients, although it is still considered high risk [1-3]. We experience in our tertiary counseling and care center that clinical decision-making in advanced CKD and pregnancy is impacted by a myriad of factors.

Dr. A. Titia Lely

Department of Obstetrics, University Medical Center Utrecht KE04.1541

NL-85500, Utrecht (The Netherlands)

a.t.lely@umcutrecht.nl 
An important aspect of (pre)pregnancy care in advanced CKD is overlooked in the literature, namely, that the counseling itself can be a complex process. Limited data and unknown risks regarding the outcome, as well as differences in risk perception, can make providing the (prepregnancy) care needed to ensure a good outcome challenging [1].

\section{Methods}

We aim to shed light on the clinical practice considerations we encounter, providing our perspective on how to deal with decision-making in daily practice. We do this by discussing the 4 main preconception counseling topics that we find can lead to discussions on the best care for a specific patient in our tertiary clinic: (1) the high risk of pregnancy complications, (2) the risk of permanent CKD deterioration due to pregnancy and subsequent shortened life expectancy, (3) changes in renal medication needed in pregnancy, and (4) assisted reproduction, genetic testing, and prenatal or preimplantation genetic diagnostics (PGD). Additionally, we reflect on how we deal with the clinical practice dilemmas we encounter in our clinics, to hopefully contribute to a broader discussion on the best prepregnancy care for CKD patients.

\section{Results}

\section{Increased Prevalence of Pregnancy Complications in CKD}

Although data are limited, and often conflicting, overall studies have shown increased risks of pre-eclampsia (odds ratio [OR] 7-14), caesarean section (OR 2-3), prematurity (OR 3-9), low birthweight (OR 2-6), and need for admittance to a neonatal intensive care unit (NICU) [4]. As displayed in Figure 1, absolute risks of an adverse outcome depend on the CKD stage and whether the patient is on dialysis or is posttransplant [5-7]. For example, for patients with advanced CKD, the pre-eclampsia rate is $\sim 50 \%$, caesarean section $\sim 70 \%$, prematurity $\sim 90 \%$, low birthweight $\sim 50 \%$, and need for NICU admission $\sim 70 \%$ $[1,5,8,9]$. One should note that the definition of (superimposed) pre-eclampsia varies between publications, and the $\sim 50 \%$ should, therefore, be interpreted with caution. Overall, fetal complications could lead to neurodevelopmental delay for the child, albeit with the improvement in neonatal care, many premature babies can lead normal lives [10].

As the chance of complications significantly increases with advancement in CKD stage (Fig. 1), ideally one would discuss planning a pregnancy while the patient is still in CKD stages 1-3 and risks are relatively low [5].

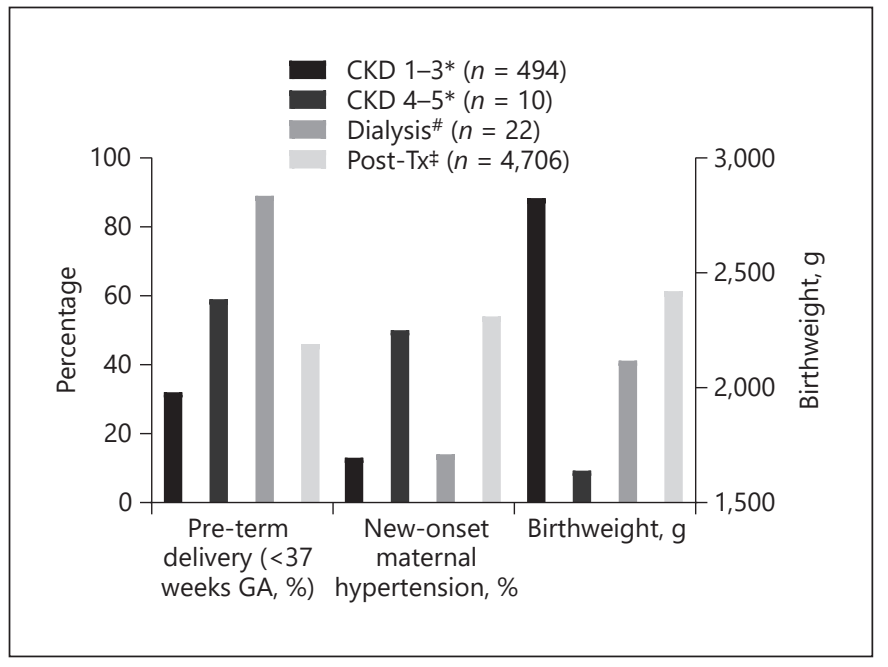

Fig. 1. Illustrative figure showing that the prevalence of maternal and fetal pregnancy complications (low birthweight, preterm delivery, need for NICU, and new-onset maternal hypertension) increases with advancement in CKD stage. Though data are limited, evidence shows that in advanced stages of CKD (intensive and nocturnal) dialysis risks are high, which decrease after a kidney transplant. Therefore, adequate timing of a pregnancy is vital. Data derived from *Piccoli et al. [5], ${ }^{*}$ Hladunewich et al. [7], and ${ }^{\ddagger}$ Deshpande et al. [6].

Risks increase once the patient is in CKD stage 4 or 5 or on dialysis $[5-7,11]$. Therefore, one could decide to postpone a pregnancy until after a kidney transplant. Deciding to postpone is not a clear-cut decision and depends on, among others, the length of the transplantation process, the risk of suboptimal kidney function posttransplant (which cannot be estimated beforehand), and the impact of a pregnancy on the graft.

Finally, although attainable with intensive dialysis schedules (e.g., nocturnal hemodialysis for $42 \mathrm{~h}$ a week), pregnancy is most high risk in dialysis patients $[6,7,11]$. Patients and physicians may face a dilemma in cases where not harming the mother might harm the (future) fetus. This dilemma arises in cases where waiting until after a transplant is not preferable, due to maternal age or long waiting lists, and one has to decide whether the fetal complications of a pregnancy on intensive dialysis can be accepted.

\section{Risk of Permanent Renal Function Deterioration due to Pregnancy and Limitations to Life Expectancy}

Next to the obstetric complications, pregnancy leads to a permanent deterioration of renal function in $6-31 \%$ of women $[4,5,12]$. This so-called CKD-shift (Fig. 1) is 
complex in etiology, although the higher filtration rate needed in pregnancy likely plays a role $[4,5,12]$. A CKDshift means that some stage 4-5 patients may need to start dialysis during or in the years after pregnancy, which they otherwise might not have to.

Even without a CKD-shift, life expectancy in patients with impaired renal function is significantly shortened; women with an eGFR (estimated glomerular filtration rate) of 15-29 have a life expectancy of $\sim 13$ years in comparison to the healthy population [13]. Thus, the maternal lifespan (or lifespan without renal replacement therapy) may not extend to her future child's adulthood. A poignant example is that of women with a renal transplant, as $12 \%$ die within 20 years after delivery (median 6 years) [14].

The long-term consequences of a CKD-shift show that clinical practice decisions in pregnancy and CKD do not only span the pregnancy itself, but also the rest of the mother's and child's life. The poor renal outcome and limited life expectancy provide a large burden, greatly impacting the quality of life for the mother, partner, and child [15]. As is the case for other chronic diseases, such as heart disease, decisions for child care, as they pertain to shortened maternal life expectancy, ought to be addressed in the preconception stage [16]. Even though maternal survival into the child's adulthood is likely for most cases, comprehensive preparations with the patient's partner and extended social network can be useful when adverse outcomes occur [16]. Further complicating this topic is that the prognosis of CKD patients has steadily increased over the past years, a trend luckily likely to continue, making it difficult to estimate the quality of life for CKD patients $10-20$ years from now.

\section{Changes in Renal Medication when Considering Pregnancy}

Fetal safety of a patient's current treatment is a factor to take into account. Within the large branches of CKD treatment (antihypertensive, immunosuppressive, and biological drugs), there are various safe drugs [1]. Nevertheless, many immunosuppressive drugs (including mycophenolate) are contraindicated in pregnancy because of teratogenicity and first-trimester losses and should, therefore, be discontinued timely $[1,17]$.

The considerations regarding maternal treatment surround whether it is advisable to discontinue certain drugs because of teratogenicity or insufficient safety data, while discontinuation could cause adverse maternal renal disease outcomes (especially posttransplant). Additionally, the unknown fetal side effects of certain drugs can render the decision even more complex, as one does not know if discontinuing a drug important for maternal care will even reduce fetal harm [1].

\section{Assisted Reproduction, Genetic Testing, and PGD}

During any preconception counseling, a topic to consider is the impaired fertility that is common in women with advanced CKD $[1,4]$. More than healthy women, patients may require ovulation induction or assisted reproduction techniques such as in vitro fertilization [4].

In assisted reproduction cases, as well as spontaneous pregnancies, genetic testing should be considered. Genetic diseases are highly prevalent in the young CKD population: $20 \%$ of all ESRD patients presenting before the age of 25 have a monogenic kidney disease [18]. A monogenic disease impacts not only the patient, for instance because different therapies might be indicated, but also her offspring, which is at risk of inheriting the kidney disease. Although not in all patients the causative genetic defect can be found, providing genetic testing opens many avenues for patients [19].

When the causative mutation is known, invasive prenatal diagnostic (PND) testing (chorion villus biopsy or amniocentesis) can be performed, with a possibility to terminate an affected pregnancy [20]. To avoid a need for invasive diagnostics (with risk of miscarriage) and termination, PGD testing has been developed for patients with a known monogenic mutation. It brings down the risk of passing on the genetic disease to the future child to $1-2 \%$ [21]. PGD entails performing genetic testing in a single cell removed from an in vitro fertilization-embryo and only transferring a genetically unaffected embryo to the uterus [21]. Furthermore, the physician should realize that genetic testing in general and PGD specifically can be time consuming (3-24 months). Thus, this is among the first topics to discuss with a CKD patient to ensure adequate genetic counseling and care.

Although surrounded with many large- and smaller scale good clinical practice considerations, which are beyond the scope of this study, the application of PND and PGD testing is widely accepted, especially in diseases that have an early onset and are severe [22]. Still, in the Netherlands, a (nationwide) committee of expert physicians and bioethicists deliberate on each new gene to ensure the decision to perform PGD testing is morally sound for that specific gene. Whether or not PND or PGD testing can be considered depends on its local availability, disease severity, and patient preference $[20,23]$. 


\section{Discussion}

As stated before, the preconception counseling in CKD can be complex, causing discussions on the best practice for a specific patient. Below, we provide our perspective on how we deal with these discussions and how we ensure adequate decision-making in our clinic.

\section{Physician Attitude, Paternalism, and Shared \\ Decision-Making}

The method of preconception counseling is essential. We feel that for adequate decision-making, the patient should be fully informed about the risks and potential complications of a pregnancy in CKD. Therefore, we apply a so-called interpretive attitude toward the physicianpatient relationship, which defines the physician as being "a counselor (...), supplying relevant information, helping to elucidate values and suggesting what medical interventions realize these values" [24]. An interpretive attitude in the physician-patient relationship does not allow for "hard paternalism" (overriding the preferences of that person), yet "soft paternalistic" approaches or directive counseling (providing information and even advising negatively) could be applied to ensure the patient has all the input needed for decision-making [24]. The exchange of ideas between a physician and a patient (shared decision-making) permits the patient an independent choice, enhancing her autonomy $[25,26]$.

\section{Reproductive Autonomy and Nonmaleficence}

Reproductive autonomy, the liberty to decide whether or not to have children, is the main principle in any discussion concerning reproduction [27]. Specifically, in every decision on pregnancy in $\mathrm{CKD}$, one has to weigh the maternal reproductive autonomy against the principle to do no harm (nonmaleficence) to the woman or the fetus $[28,29]$.

In principle, we regard the patient's autonomy as paramount in every medical decision. We apply the concept of "relational autonomy," where one includes contextual factors, such as the patient's emotional background, and social and financial factors [27]. An important contextual factor is the patient's partner, who can contribute in many ways to the patient's autonomy and the decisions regarding pregnancy. This leads to an open conversation whereby the patients feel free to express themselves.

Even though CKD is a risk factor for suboptimal pregnancy outcome, in the majority of cases, we feel that the desire to not harm the mother or the fetus does not outweigh the maternal reproductive autonomy. Underscor- ing this is the notion that pregnancy is inherently a highrisk situation, for example, $3-5 \%$ of all pregnancies are complicated by pre-eclampsia, regardless of maternal comorbidity [30]. Therefore, the preconception counseling is aimed at the patient understanding the potential risks and the physician and patient working together to minimize these risks as much as possible.

Yet, when there is a need for assisted reproduction, we find that considerations of not harming the mother and fetus are more relevant. That is to say, there is a difference between caring for a patient when she becomes pregnant naturally and assisting in initiating a pregnancy that puts the mother and the fetus at high risks. In such cases, we argue that the physician can justifiably act more paternalistically, since he or she is actively assisting (instead of passively allowing) a situation which one highly suspects will harm a future fetus $[28,29]$.

\section{Multidisciplinary Care}

One of the ways we ensure adequate decision-making in our tertiary care facility is by providing multidisciplinary care. We offer a multidisciplinary outpatient clinic where patients are counseled by a nephrologist and specialized maternal-fetal medicine specialist. A clinical geneticist specialized in hereditary kidney disease consults on genetic testing, PND, and PGD, if applicable. The team also confers with fertility specialists, pathologists, ethicists, and anesthesiologists to gain insights into technical care matters related to pregnancy, as well as ethical issues that may arise. Furthermore, in cases of assisted reproduction or PGD, the team is advised by local and national expert committees consisting of physicians and medical ethicists.

In conclusion, due to the advances in nephrological, fertility, and obstetric care, patients with advanced CKD have a myriad of choices regarding pregnancy. They should be counseled on the available factual information regarding increased pregnancy and renal complications, and their long-term impact, including a limited life expectancy. Furthermore, the options regarding assisted reproductive technology, genetic testing, PND, and PGD should be discussed. However, these topics and the decisions they entail can cause deliberations between physicians, and with the patient, especially since data on these issues are limited. We provide our perspective on how to deal with these situations, namely, by ensuring an interpretive attitude in the physician-patient relationship and shared decision-making, additionally deliberating on clinical practice dilemmas in a multidisciplinary setting and, if needed, with input from an expert committee. 


\section{Statement of Ethics}

Because this study did not involve human subjects, no ethical approval was required as per Dutch law.

\section{Funding Sources}

This study was supported by the Dutch Kidney Foundation grant $15 \mathrm{OP} 14$ to A.M. van Eerde.

\section{Disclosure Statement}

The authors declare that they have no relevant financial interests.

\section{References}

1 Wiles KS, Nelson-Piercy C, Bramham K. Reproductive health and pregnancy in women with chronic kidney disease. Nat Rev Nephrol. 2018 Mar;14(3):165-84.

2 Hall M. Pregnancy in women with CKD: a success story. Am J Kidney Dis. 2016 Oct; 68(4):633-9.

3 Nevis IF, Reitsma A, Dominic A, McDonald S, Thabane L, Akl EA, et al. Pregnancy outcomes in women with chronic kidney disease: a systematic review. Clin J Am Soc Nephrol. 2011;6(11):2587-98.

4 Zhang J-J, Ma X-X, Hao L, Liu L-J, Lv J-C, Zhang $\mathrm{H}$. A systematic review and meta-analysis of outcomes of pregnancy in CKD and CKD outcomes in pregnancy. Clin J Am Soc Nephrol. 2015 Nov;10(11):1964-78.

5 Piccoli GB, Cabiddu G, Attini R, Vigotti FN, Maxia S, Lepori N, et al. Risk of adverse pregnancy outcomes in women with CKD. J Am Soc Nephrol. 2015;26(8):2011-22.

6 Deshpande NA, James NT, Kucirka LM, Boyarsky BJ, Garonzik-Wang JM, Montgomery RA, et al. Pregnancy outcomes in kidney transplant recipients: a systematic review and meta-analysis. Am J Transplant. 2011;11(11): 2388-404.

7 Hladunewich MA, Hou S, Odutayo A, Cornelis T, Pierratos A, Goldstein M, et al. Intensive hemodialysis associates with improved pregnancy outcomes: a Canadian and United States cohort comparison. J Am Soc Nephrol. 2014;25(5):1103-9.

8 Wiles KS, Bramham K, Vais A, Harding KR, Chowdhury P, Taylor CJ, et al. Pre-pregnancy counselling for women with chronic kidney disease: a retrospective analysis of nine years' experience. BMC Nephrol. 2015 Mar;16:28.

9 Bramham K, Seed PT, Lightstone L, NelsonPiercy C, Gill C, Webster P, et al. Diagnostic and predictive biomarkers for pre-eclampsia in patients with established hypertension and chronic kidney disease. Kidney Int. 2016 Apr; 89(4):874-85.
10 Pierrat V, Marchand-Martin L, Arnaud C, Kaminski M, Resche-Rigon M, Lebeaux C, et al. Neurodevelopmental outcome at 2 years for preterm children born at 22 to 34 weeks' gestation in France in 2011: EPIPAGE-2 cohort study. BMJ. 2017 Aug;358:j3448.

11 Piccoli GB, Cabiddu G, Daidone G, Guzzo G, Maxia S, Ciniglio I, et al. The children of dialysis: live-born babies from on-dialysis mothers in Italy - an epidemiological perspective comparing dialysis, kidney transplantation and the overall population. Nephrol Dial Transplant. 2014;29(8):157886.

12 Piccoli GB, Cabiddu G, Attini R, Gerbino M, Todeschini P, Perrino ML, et al. Outcomes of pregnancies after kidney transplantation. Transplantation. 2017;101(10):2536-44.

13 Neild GH. Life expectancy with chronic kidney disease: an educational review. Pediatr Nephrol. 2017 Feb;32(2):243-8.

14 van Buren M, Lely T, van de Wetering J. Essential issues for pregnancy counseling in renal transplant women. Transplantation. 2018 Jun;102(6):e254.

15 Golics CJ, Basra MKA, Salek MS, Finlay AY. The impact of patients' chronic disease on family quality of life: an experience from 26 specialties. Int J Gen Med. 2013;6:787-98.

16 Simpson LL. Preconception considerations. Semin Perinatol. 2014 Aug;38(5):236-9.

17 Skorpen CG, Hoeltzenbein M, Tincani A, Fischer-Betz R, Elefant E, Chambers C, et al. The EULAR points to consider for use of antirheumatic drugs before pregnancy, and during pregnancy and lactation. Ann Rheum Dis. 2016;75(5):795-810.

18 Vivante A, Hildebrandt F. Exploring the genetic basis of early-onset chronic kidney disease. Nat Rev Nephrol. 2016 Mar;12(3):13346.

19 Groopman EE, Rasouly HM, Gharavi AG. Genomic medicine for kidney disease. Nat Rev Nephrol. 2018 Feb;14(2):83-104.
20 Skirton H, Goldsmith L, Jackson L, Lewis C, Chitty L. Offering prenatal diagnostic tests: European guidelines for clinical practice. Eur J Hum Genet. 2014;22(5):580-6.

21 Harton GL, De Rycke M, Fiorentino F, Moutou C, SenGupta S, Traeger-Synodinos J, et al. ESHRE PGD consortium best practice guidelines for amplification-based PGD. Hum Reprod. 2011 Jan;26(1):33-40.

22 Traeger-Synodinos J. Pre-implantation genetic diagnosis. Best Pract Res Clin Obstet Gynaecol. 2017 Feb;39:74-88.

23 van Eerde AM, Krediet CTP, Rookmaaker MB, van Reekum FE, Knoers NVAM, Lely AT. Pre-pregnancy advice in chronic kidney disease: do not forget genetic counseling. Kidney Int. 2016 Oct;90(4):905-6.

24 Emanuel EJ, Emanuel LL. Four models of the patient-physician relationship. J Am Med Assoc. 1992;267(16):2221-6.

25 Quill TE, Brody H. Physician recommendations and patient autonomy: finding a balance between physician power and patient choice. Ann Intern Med. 1996 Nov; 125(9):763-9.

26 Harris J, Keywood K. Ignorance, information and autonomy. Theor Med Bioeth. 2001 Sep; 22(5):415-36.

27 Gaucher N, Payot A. Focusing on relationships, not information, respects autonomy during antenatal consultations. Acta Paediatr. 2017 Jan;106(1):14-20.

28 Laufer-Ukeles P. Reproductive choices and informed consent: fetal interests, women's identity, and relational autonomy. Am J Law Med. 2011;37(4):567-623.

29 Murtagh GM. Ethical reflection on the harm in reproductive decision-making. J Med Ethics. 2007 Dec;33(12):717-20.

30 Abalos E, Cuesta C, Grosso AL, Chou D, Say L. Global and regional estimates of preeclampsia and eclampsia: a systematic review. Eur J Obstet Gynecol Reprod Biol. 2013 Sep; 170(1):1-7. 